\title{
Are new agents needed to treat RA?
}

T herapy for rheumatoid arthritis (RA) was transformed a decade ago by the introduction of biologic agents, and specifically agents that target the inflammatory cytokine tumor necrosis factor (TNF). Since the initial approval of etanercept, and shortly thereafter infliximab, three additional TNF-neutralizing antibodies (adalimumab, certulizumab pegol and golimumab) have been approved, as well as the antiT-cell (and/or dendritic cell) therapy, abatacept, and the B-cell-depleting antibody, rituximab. The interleukin (IL)-1 antagonist, anakinra, was also approved for the treatment of RA, but is not commonly used because of the low efficacy of this agent. Approved in some parts of the world, but not yet in the US, is an additional agent, the IL-6 receptor antagonist, tocilizumab.

The availability of one or another of this bouquet of biologic agents, frequently used in combination with more classic chemical DMARDs, has given rheumatologists new and very potent means to treat patients with this chronic inflammatory condition, and has greatly changed patients' expectations. It is safe to say that, fueled by direct Pharma-to-consumer advertising, patients with RA now expect to lead normal lives, whereas previously the expectation of therapy was a reduction of pain, although ongoing inflammation, diminished physical function, work disability and a shortened life expectancy were accepted as the normal course of events. The perceived impact of biologic agents has been so great that many no longer view RA as an unmet medical need. As to whether current patient expectations are realistic and whether additional antirheumatic agents are needed are open questions; the intense activity in the pharmaceutical industry in this area suggests that they believe there remains a gap in the market.

Despite the obvious impact of biologic agents, a number of issues should be considered when asking whether there is a need for additional agents to treat RA. The first is whether these agents actually can induce a sustained treatment-free remission, the obvious goal of therapy for RA. A recent report indicates that prolonged therapy with classic chemical DMARDs can achieve DMARD-free sustained remission in only $9.4-15 \%$ of patients with RA (van der Woude, D. et al. Arthritis Rheum. 60, 2262-2271 [2009]). Using far more relaxed criteria of 'remission' that do not require prolonged absence of signs and symptoms of inflammation without DMARD therapy, most studies still suggest that 'remission' with biologic therapy is not common (Emery, P. et al. Arthritis Rheum. 60, 22712283 [2009]; Keystone, E. C. et al. Ann. Rheum. Dis. 68, 789-796 [2009]).
If remission is the goal, then clearly this has not been uniformly achieved with the current biologic agents. Of course, it could be argued that prolonged treatment-free remission is too high a bar to gauge success of therapy, and that achievement of a low disease activity state with continued treatment is sufficient. Patients with RA might not agree with this, and insurers and other payers of health care might object. Moreover, the fact remains that current data indicate that even the achievement of a low disease activity state with therapy is not uniform with the current biologic agents. There could be a number of reasons for this, including initiation of therapy too late in the course of disease, insufficient length of therapy, or inappropriate co-therapy. Some have even argued that, as anti-cyclic citrullinated peptide (CCP) antibodies occur before the onset of signs and symptoms of RA, preemptive therapy in individuals who are anti-CCP positive but lack manifestations of inflammatory arthritis might be necessary to prevent the cascade of events that results in the clinical appearance of RA. Whether this is practical remains to be seen. With current approaches to therapy with biologic agents that are associated with a lack of uniform induction of even a low disease activity state on persistent treatment, it is difficult to accept that there is no more progress to be made in achieving the ultimate goal of RA therapy-namely true remission.

In addition, the current biologic agents are associated with a number of adverse effects that make their persistent administration concerning. Especially worrisome are the concerns about various infectious complications and, with some biologic agents, the suspicion that they are associated with increased risks of malignancies; therefore, identifying new agents that are associated with a greater frequency of remission induction and less concerns about unwanted adverse effects remains an important goal.

One possible explanation for the failure to achieve remission routinely with current biologic agents relates to the targets of these products: they all target inflammatory cytokines (such as TNF, IL-1 and IL-6) or cells involved in immune inflammation ( $\mathrm{T}$ cells and/or dendritic cells and B cells). In other words, these agents are directed toward inflammatory processes, but not necessarily disease causation. One could argue that in the absence of an approach that actually eradicates the cause of RA, 'cure' with a resultant prolonged treatment-free remission in signs and symptoms of inflammatory disease is very unlikely. Of course, dysregulation of inflammatory cytokines or B-cell or T-cell activation could lead to secondary abnormalities that perpetuate rheumatoid

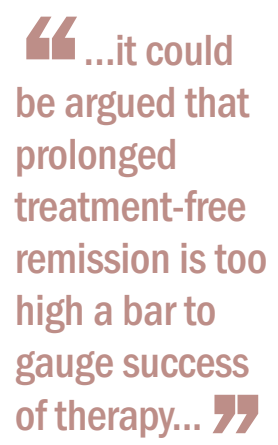

Peter E. Lipsky is the Editor-in-Chief of Nature Reviews Rheumatology.

Competing interests The author declares no competing interests. 
inflammation, such as production of additional cytokines or perturbations of the function of bystander cells, such as regulatory $\mathrm{T}$ cells, synoviocytes or osteoclasts; however, much of these effects must be considered as secondary or amplifying processes that might be essential for the full clinical picture of RA, but are unlikely to be primary initiating events.

The classic pathogenic view of RA remains that of a disease in which an inciting stimulus initiates a pathologic response in genetically susceptible individuals. Many genes have been identified that contribute risk for RA or greater severity of rheumatoid inflammation, and together are beginning to complete the picture of the genetically susceptible host; however, research on the inciting triggers has revealed the role of cigarette smoking, but little else. Indeed, even cigarette smoking seems to work largely in a genetic context to increase the risk of producing anti-CCP antibodies. Thus, thinking about targets that might truly have the potential to be curative by blocking etiologic processes in RA is challenging.

Years ago when alleles of the HLA-DR locus were identified as important risk factors for RA, various attempts to target either the antigen presenting capability of the HLA-DR molecule or the activity of antigen presenting cells or $\mathrm{CD} 4^{+}$helper $\mathrm{T}$ cells were undertaken, as the only known function for HLA-DR was in antigen presentation to $\mathrm{CD} 4^{+}$helper T cells. Most of these trials were carried out in patients with advanced disease and were uninformative, and were largely abandoned when trials of depleting anti-CD4 antibodies (along with antibodies to CD5, CD7 and CD52) failed to show benefit. More successful recent trials with abatacept, which targets $\mathrm{T}$ cell co-stimulation and/or dendritic cell function, have, however, clearly demonstrated a role for T-cell activation in rheumatoid inflammation. It might be time, therefore, to reconsider additional approaches to antigen-specific T-cell activation employing the insights that have been learned over the past decade in clinical trial design in RA.
A greater understanding of the salient activities responsible for the efficacy of the currently employed biologic agents might also aid in the development of better therapeutic agents. Currently available biologic agents target remarkably pleiotropic cells or cytokines (all $\mathrm{CD} 20^{+} \mathrm{B}$ cells, all co-stimulatory signals to $\mathrm{CD} 4^{+} \mathrm{T}$ cells, and all activities of TNF or IL-6). Undoubtedly, a small subset of these activities might be important in the effect on rheumatoid inflammation, whereas an only partially overlapping set of activities might be involved in contributing to essential innate or adaptive immune responses. Sorting out these pathways and cellular subsets might permit identification of a more precise activity of these cytokines or cells that contributes to rheumatoid inflammation but that is less essential for host defense against infection or tumor surveillance, so that blockade would produce fewer untoward effects.

Alternatively, a greater effort at developing the tools to employ 'personalized' medicine might be useful in an attempt to match the patient with the most appropriate biologic therapy. Numerous efforts to identify patients who respond well to specific biologic agents have begun to yield profiles that might allow more personalized use of these agents in individual patients, but much more work needs to be done.

Finally, it might be necessary to develop new targets to suppress or cure RA more effectively. This issue of Nature Reviews Rheumatology includes a review outlining the current landscape of RA therapy (available therapeutics, accepted principles of RA management and some controversies in this field) and a series of articles in which experts in specific areas of biologics research identify the new target that they feel might generate the most effective 'next generation' of therapy for patients with RA. These discussions should provide unique insights, not only into the current status of RA therapy, but also what the future might hold as we look for ways to cure patients with this chronic inflammatory disease.

doi:10.1038/nrrheum.2009.197 